$11 / 30 / 2000$

AIAA CONFERENCE, RENO NEVADA, JANUARY 8-11, 2001

\title{
PRELIMINARY PHOTON PRESSURE MEASUREMENTS USING A SOLAR SIMULATOR
}

Authors: P. A. Gray, D.L. Edwards, M. R. Carruth, Jr.

\section{ABSTRACT}

Initial proof of concept photon momentum measurements are reported verifying photon pressure on a simulated sail material can be measured under high vacuum conditions using a full spectrum solar simulator and a vacuum compatible force measurement system. Second order effects such as sample reflectivity, beam uniformity, radiometric flux measurement accuracy, and the optical system have been accounted for in evaluating these measurements.

\section{INTRODUCTION}

The concept of using photon pressure for propulsion has been around for some time. The photon pressure that is created when light hits a surface has been postulated since the advent of quantum physics. Lebedev, and Nicholes and Hull (Ref 1 and 2) made the first experimental measurements of this force in the early 1900's. These measurements used a torsion balance to measure the light pressure from a concentrated light source. Outgassing and convection of the gases surrounding the vanes that were used to measure the light pressure complicated these measurements. Some attempts were made to reduce this effect by lowering the pressure surrounding the test apparatus and by canceling out these forces by heating both vanes on the torsion balance. Also, methods were used where the vanes were only intermittently exposed to light to reduce the effect of gas heating. No significant experimental work has been done since these early experiments in the early 1900's.

Since that time, the state of the art has greatly increased in the field of vacuum technology. Today pressures in the 1e-7 to 1e-8 torr range are easily achievable. There have also been technological advances making it possible to measure very small forces in vacuum on the order of $1 \times 10^{-8}$ micronewtons

\section{EXPERIMENTAL SETUP}

All of the hardware for this experiment is standard off the shelf vacuum hardware and laboratory instrumentation. The photon pressure measurements are made in an ion pumped vacuum system with a working pressure of 1 e-7 torr. On top of the ion pump is an 8 inch manually operated vacuum gate valve. On top of the gate valve is a 10 inch conflat ( 7.75 inch ID) 6 way vacuum cross. The cross has 
a windowed view port and a 10 inch UV enhanced quartz window. Currently the windowed view port is being used to illuminate the sample. This was done to avoid reconfiguring the chamber during the initial proof of principle stage of the experiment. A vacuum compatible force measuring device is used to measure the force created when the 2.5 sun beam from the Spectrolab, X25 solar simulator hits the 4.75 inch diameter target of cleaned and baked aluminum foil. The sample is suspended from the balance using a long nichrome wire. The force measuring device has a theoretical resolution of 0.01 micronewtons. The 6 inch diameter X25 beam passes through the glass view port and onto a 45 degree over-coated aluminum first surface mirror that is facing upward. This causes the sample to appear to decrease in weight as a result of the photons hitting the aluminum foil sample from the bottom. See Figure 1.0 for the experiment layout.

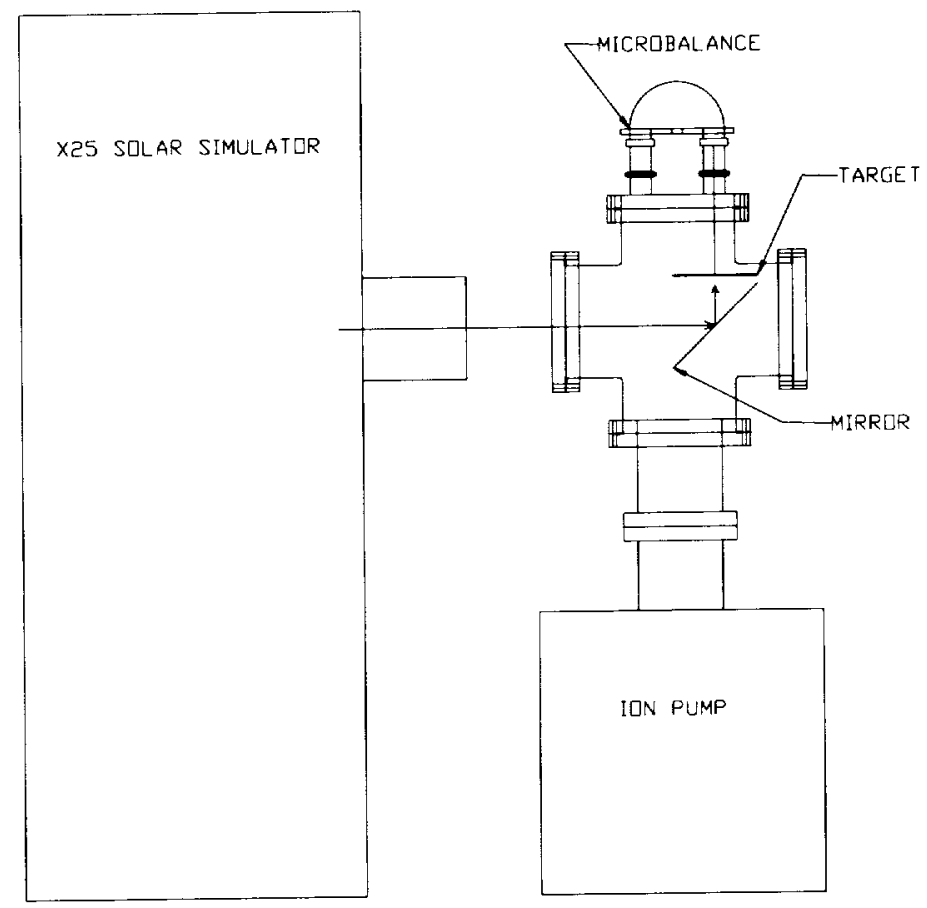

Figure 1.0 
The final value of the calculated force is.

$$
F_{c}=.231 \text { micronewtons }
$$

The measured force $\left(F_{m}\right)$ based on an average of 13 data points is

$$
F_{m}=.217 \text { micronewtons. }
$$

The theoretical maximum $\left(F_{t}\right)$ is:

$$
F_{T}=.251 \text { micronewtons }
$$

The actual test data is within $93.6 \%$ of the estimated value. The standard deviation is $7.2 \%$ of the average of the 13 data points. The estimated uncertainty of the intensity measurements from the hand held thermopile is $+-5 \%$ of the reading. The thermopile was calibrated using an NIST traceble spectroradiometer.

\section{CONCLUSIONS}

Modern methods can be successfully used to measure photon momentum transfer to sail materials. The deviation from the estimated values was less than ten percent. With further improvement in techniques and more detailed and accurate characterization of the losses involved it should be possible to improve on this data. No attempt was made to estimate the loss of efficiency due to a non-specular sample and no attempt was made to estimate the effect of reemmision of light from the back side of the sample. Both of these contributions are considered to be second or third order effects. The data would tend to confirm that this is the case.

References:

1. Lebedev, P., "The Physical Causes of Deviation from Newton's Laws of Gravitation", Astrophysical Journal, 10, 155-161, 1902.

2. Nichols, E. F. and Hull, G. F., "The Pressure Due to Radiation", Physical Review, 17, 26-50, 1903. 